\title{
A Dialética da Opinião Pública: \\ efeitos recíprocos da política pública e da opinião pública em sociedades democráticas contemporâneas
}

\begin{tabular}{c}
\hline Michael Howlett \\
Departamento de Ciência Política \\
Simon Fraser University - Canadá \\
\hline \hline
\end{tabular}

\section{Resumo}

Este artigo analisa o papel desempenhado pela opinião pública em cada estágio do processo de elaboração de políticas públicas. Através da desagregação desse processo em uma seqüência de etapas, ele revela se e quando a opinião pública o afeta, e onde ele dá significância a outras influências. Como conclusão, o artigo sublinha a natureza dialética da relação entre opinião pública e políticas públicas.

Palavras-chave: opinião pública, políticas públicas, processo de elaboração de políticas, governança.

Abstract

This paper examines the role played by public opinion at each stage of the policy process. By disaggregating policy making into a sequence of steps, it reveals if and where public opinion affects policy-making and where it cedes significance to other policy. As conclusion the paper underlines the dialectical nature of the public opinion and public policy relationship.

Key words: public opinion, public policy, policy making process, governance. 
Que imagem da democracia nos leva a atribuir à opinião pública um lugar central na teoria política? ...Antes de investirmos as energias de uma geração de cientistas políticos na pesquisa de opinião pública, não seria interessante tentar testar a validade das proposições subjacentes às relações da opinião pública com o que acontece no mundo à nossa volta?

Schattschneider, E. E. The Semisovereign People. A Realist's View of Democracy in America. New York, Holt, Rinehart and Winston, 1960, p. 131.

\section{Apresentação \\ A natureza normativa de muitas das pesquisas sobre a relação existente entre as políticas públicas e a opinião pública}

Embora muitos trabalhos nas últimas décadas tenham definido a relação entre a opinião pública e a formação de políticas públicas em sociedades democráticas como uma relação complexa, persiste uma tendência a considerar essa relação como simples, direta e linear. ${ }^{1}$ Isto é, pelo menos desde os primeiros trabalhos de V. O. Key, E. E. Schattschneider e Bernard Berelson nos anos 50 e 60, importantes cientistas políticos, e também outros, tem continuamente achado que há muito pouca, ou mesmo nenhuma, ligação entre a opinião pública e os resultados políticos. ${ }^{2}$ Apesar disso, essa descoberta tem sido retomada e refeita em muitos estudos, como se os pesquisadores estivessem insatisfeitos com a resposta. ${ }^{3}$ Como sugeriu Schattschneider, isso sem dúvida se deve às noções sinceras, mas às vezes simplistas, da democracia dos analistas que apóiam a idéia de um "governo para o povo" e ficam constrangidos de que isso pode não ser alcançado através do "governo pelo povo".

A natureza normativa e ideológica de muitas das discussões sobre o tema aparece claramente nos títulos e termos utilizados para descrever muitos resultados. Recentemente, isso pode ser visto nos excelentes estudos históricos comparativos de Joel Brooks que, descobrindo a pouca relação entre opinião pública e a formação de políticas, chama o fenômeno de "frustração democrática", sugerindo que ele resulta de um problema quando o sistema de políticas não reage de maneira apropriada ao sistema

\footnotetext{
${ }^{1}$ Ver Luttbeg, Norman R., Where We Stand on Political Linkage. In N. R. Luttbeg, (org) Public Opinion and Public Policy: Models of Political Linkage, Itasca, F. E. Peacock, 1981: 455-62 e Shapiro, Robert Y. e Lawrence R. Jacobs, The Relationship Between Public Opinion and Public Policy: A Review. In S. Long (org), Political Behavior Annual, Boulder, Westview, 1989

${ }^{2}$ Ver V. O. Key Jr, Public Opinion and American Democracy, New York, Knopf, 1967, Schattschneider, E. E., The Semisovereign People. A Realist's View of Democracy in America, New York, Holt, Rinehart and Winston, 1960, e Bernard Berelson, Democratic Theory and Public Opinion, Public Opinion Quarterly 16 1952: 313-330.

${ }^{3}$ Ver, por exemplo, Monroe, Alan D., Consistency Between Public Preferences and National Policy Decisions, American Politics Quarterly 7 (1) 1979: 3-19 e, mais recentemente, Page, Benjamin I. e Robert Y. Shapiro, The Rational Public: Fifty Years of Trends in American Policy Preferences, Chicago, The University of Chicago Press, 1992.
} 
democrático. ${ }^{4}$ Mas tanto os processos das políticas quanto os processos democráticos são muito mais complexos do que sugerem as teorias da ligação linear. ${ }^{5}$ Ou seja, a democracia significa mais do que o governo pela multidão e teóricos pelo menos desde Edmund Burke assinalou as complexas noções de representação popular que os governos democráticos contêm. ${ }^{6}$ De maneira semelhante, os estudiosos da formação de políticas desde Harold Lasswel chamaram atenção para a natureza complexa dos atores, idéias, instituições e interesses que se combinam para produzir políticas. ${ }^{7}$

A análise do papel da opinião pública nos governos democráticos contemporâneos requer uma análise nuançada das realidades dos processos de governança democrática e da formação de políticas. Embora a preocupação com a soberania popular seja louvável, como a citação de Schattschneider sugere, especulações teóricas devem ser temperadas por fatos empíricos a fim de que a natureza da relação existente entre a opinião pública e as políticas públicas seja adequadamente descrita e compreendida.

\section{Os Efeitos da Opinião Pública na Formação de Políticas (1): o modelo dos efeitos diretos e lineares}

O modelo mais simples das relações existentes entre a opinião pública e a formação de políticas públicas é o que concebe o governo simplesmente como uma máquina de formação de políticas - processando diretamente os sentimentos populares em decisões de política pública e estratégias de implementação. Como tem sido repetidamente observado nos estudos sobre o tema no último meio século nos Estados Unidos e em outros lugares, porém, essa descrição é problemática por muitas razões. ${ }^{8}$

Primeiro, como os pensadores clássicos observaram há muito tempo, supõe-se que a opinião pública tem um caráter concreto, quase permanente, que pode ser agregado com facilidade em posições políticas coerentes. Inúmeros estudos, a partir

\footnotetext{
${ }^{4}$ Brooks, Joel E., Democratic Frustration in the Anglo-American Polities: A Quantification of Inconsistency Between Mass Public Opinion and Public Policy, Western Political Quarterly 38 (2) 1985: 250-261; Brooks, Joel E., The Opinion-Policy Nexus in France: Do Institutions and Ideology Make a Difference?, The Journal of Politics 49 (2) 1987: 465-480; e Brooks, Joel E., The Opinion-Policy Nexus in Germany, Public Opinion Quarterly 54 (3) 1990: 508-529. Mais recentemente, ver Petry, François, The Opinion-Policy Relationship in Canada, The Journal of Politics 61 (2) 1999: 540-550.

${ }^{5}$ Buckley, Walter, Society as a Complex Adaptive System. In W. Buckley (org) Modern System Research for the Behavioral Scientist, Chicago, Aldine, 1968: 490-513; Gell-Mann, Murray, Complexity and Complex Adaptive Systems. In J. A. Hawkins e M. Gell-Mann (orgs) The Evolution of Human Languages, Redwood City, Addison-Wesley, 1992: 3 18; Jervis, Robert, Complexity and the Analysis of Political and Social Life, Political Science Quarterly 112 (4) 1997-98: 569-594; La Porte, Todd R. (org) Organized Social Complexity: Challenge to Politics and Policy, Princeton, Princeton University Press, 1975: 3-21.

${ }^{6}$ Birch, Anthony H., Representation, New York, Praeger, 1972.

${ }^{7}$ Ver Heclo, Hugh, Ideas, Interests and Institutions. In L. C. Dodd e C. Jillson (orgs) The Dynamics of American Politics: Approaches and Interpretations, San Francisco, Westview, 1994: 366-392.

${ }^{8}$ Erikson, Robert S., Norman R. Luttbeg e Kent L. Tedin (orgs), American Public Opinion. New York, John Willey and Sons, 1980, e Erikson, Robert S., Gerald C. Wright e John P. McIver, Political Parties, Public Opinion and State Policy in the United States, American Political Science Review 83 (3) 1989: 729-743.
} 
pelo menos de Rousseau, porém, sublinharam a natureza vaga, abstrata e transitória da opinião pública, e também as dificuldades de agregar o murmúrio da vontade coletiva, como dizia Rousseau, em prescrições políticas universalmente endossadas. ${ }^{9}$ E muitos pesquisadores nas áreas de opinião e políticas públicas observaram a maneira como essas dificuldades se multiplicam à medida em que as questões científicas, assim como as complexas questões legais, vieram a dominar a formação de políticas nas sociedades contemporâneas, aumentando o divórcio entre os discursos sobre políticas e a opinião pública. ${ }^{10}$

Este é, porém, apenas o primeiro dos principais problemas lógicos e empíricos encontrados pelas teorias da relação direta da representação democrática. Isto é, mesmo supondo que os sentimentos públicos pudessem chegar diretamente e de forma consistente aos formadores de políticas, o modelo tem que enfrentar outros problemas e esses são terminais. De um lado, o modelo atribui muito pouca autonomia aos governos. Exatamente como os governos obtêm informações e como as processam não é considerada uma questão principal, exceto na medida em que esses processos bloqueiam a transformação dos sentimentos populares em ação governamental. Mas os processos pelos quais as agendas governamentais são estabelecidas e as opções de política formuladas estão longe de ser simples e não problemáticos. Numerosos estudos salientaram a natureza complexa da construção de políticas e as múltiplas interpretações concorrentes da realidade social e as respostas políticas potenciais a questões sociais que os governos enfrentam ao contemplar a ação. ${ }^{11}$ Outros ainda sublinham a complexa natureza dos processos de tomada de decisão pelo governo que não se adequam às simples formulações do modelo da relação linear. ${ }^{12}$ Finalmente, o modelo também considera não problemáticas as várias ligações que existem entre a execução da política e a publicação de decisões políticas e pronunciamentos. Uma vez mais, extensa literatura na área da administração pública sublinha a complexidade dos processos de implementação e supera a noção de que as decisões governamentais são simplesmente

\footnotetext{
${ }^{9}$ Ver Jean-Jacques Rousseau, The Social Contract and Discourses, Londres, J. M. Dent, 1973. A relevância do trabalho de Rousseau pelos pesquisadores de opinião no início do século XX. Ver Lowell, A. Lawrence, Public Opinion and Popular Government, New York, David McKay Company, 1926.

${ }^{10}$ Ver Pollock, Philip H., Stuart A. Lilie e M. Elliot Vittes, Hard Issues, Core Values and Vertical Constraint: The Case of Nuclear Power, British Journal of Political Science 23 (1) 1989: 29-50, e Torgerson, Douglas, Power and Insight in Political Discourse: Post-Positivism and Policy Discourse, in L. Dobuzinskis, M. Howlett e D. Laycock, orgs, Policy Studies in Canada: The State of the Art, Toronto, University of Toronto Press, 1996: 266-298.

${ }^{11}$ Holzner, Burkhart, e John H. Marx, Knowledge Application: The Knowledge System in Society, Boston, Allyn and Bacon, 1979; Schneider, Joseph W., Social Problems Theory: The Constructionist View, Annual Review of Sociology 11 1985: 209-229; Samuels, Warren J., 'Truth' and 'Discourse' in the Social Construction of Economic Reality: Na Essay on the Relation of Knowledge to Socioeconomic Policy, Journal of Post-Keynesian Economics 13 (4) 1991: 511-524; e Livingston, Steven G., Knowledge Hierarchies and the Politics of Ideas in American International Commodity Production, 12 (3) 1992: 223-242.

${ }_{12}$ Ver Forester, John, Bounded Rationality and the Politics of Muddling Through, Public Administration Review 44 (1) 1984: 23-31; Lindblom, Charles E., e D. K. Cohen, Usable Knowledge: Social Science and Social Problem Solving, New Haven, Yale University Press, 1979; Weiss, Carol H., Using Social Research in Public Policy Making, Lexington, Lexington Books, 1977.
} 
executadas de modo neutro e 'objetivo' pelos seus funcionários. ${ }^{13}$ Não é de surpreender que essas dificuldades com o modelo simples dos efeitos diretos da opinião pública tenham levado estudiosos da relação opinião pública-política pública a desenvolver modelos mais refinados que consideram algumas dessas questões. Ainda que alguns proponham visões gerais alternativas sobre a natureza da relação opinião-políticas, muitos podem ser considerados como tentativas de resgatar modelos lineares simples pela alteração de aspectos apenas secundários do modelo geral.

O conhecido modelo do ciclo de atenção às questões de Anthony Downs é um bom exemplo de um modelo de relação linear que leva em consideração a natureza vaga e transitória da opinião pública em questões sobre políticas específicas. Do modo como aparece em seu artigo de 1972 sobre a formação de políticas sobre o meio-ambiente, na visão de Downs, a formação de políticas públicas em muitas áreas da vida social tende a girar em torno de questões específicas que capturam momentaneamente a atenção pública, resultando em demandas por ação governamental. Entretanto, ele reconheceu explicitamente e adicionou ao modelo a noção de que esses problemas desapareceriam de vista à medida que sua complexidade ou intratabilidade fossem percebidas. Em suas palavras:

... a atenção do público raramente permanece firmemente focada sobre
qualquer questão doméstica por muito tempo - mesmo quando envolve um
problema de importância crucial para a sociedade. Em vez disso, um "ciclo
de atenção a questões" sistemático parece influenciar fortemente as
atitudes e comportamento do público em relação à maioria dos problemas
domésticos centrais. Cada um desses problemas repentinamente surge
como predominante, assim permanece por algum tempo e então - ainda
que não resolvido - gradualmente desaparece do centro da atenção
pública. ${ }^{14}$

A idéia de um ciclo sistemático de atenção a questões na formação de decisões políticas ganhou muita atenção nos anos seguintes e o trabalho de Downs é freqüentemente citado como modelo de explicação das ligações entre a opinião pública e a política pública. ${ }^{15}$ Permanecem, no entanto, vários problemas significativos, mesmo com esta versão 'melhorada' do modelo da relação direta.

Em primeiro lugar, ele contém os mesmos problemas que os modelos mais simples em termos dos supostos insustentáveis em relação aos processos de formação

\footnotetext{
${ }^{13}$ A literatura é extensa demais para ser aqui citada. Boas revisões podem ser encontradas em Allison, Graham T., e Morton Halperin, Bureaucratic Politics: A Paradigm and Some Policy Implications, World Politics 24 (Suplemento) 1972: 40-79; Page, Edward C., Political Authority and Bureaucratic Power: A Comparative Analysis, Brighton, Sussex, Wheatsheaf, 1985, e Partridge, P. H., Na Evaluation of Bureaucratic Power, Public Administration (Australia) 33 1974: 99-124.

${ }^{14}$ Downs, Anthony, Up and Down with Ecology - the 'Issue-Attention Cycle', The Public Interest 28 1972: 38-50. A citação é da página 38

15 Ver, por exemplo, Dearing, James W., e James W. e Everett M. Rogers, Agenda Setting, Thousand Oaks, Sage Publications, 1996.
} 
de políticas no governo. Ou seja, atribuir um caráter diferente, cíclico, à opinião pública não altera qualquer das outras suposições sobre a natureza linear do processamento das informações, da tomada de decisões e da administração por parte do governo. Em segundo, a hipótese original de Downs era bem vaga. Não é claro, por exemplo, se o ciclo se aplica a todos os problemas políticos ou apenas ao subconjunto de "todos os principais problemas sociais", como sugerido mais adiante no artigo (p. 41). ${ }^{16}$ Terceiro, uma vez mais, estudos empíricos não encontraram qualquer evidência da existência dos ciclos de Downs. ${ }^{17}$

Uma conclusão razoável com base na discussão acima é que quaisquer que sejam os efeitos da opinião pública sobre as políticas, eles não são diretos. Como muitos estudiosos dessa relação observaram, isso abre diversas possibilidades. Uma é que a opinião pública não tem qualquer efeito, possibilidade descartada pelos muitos estudos empíricos que encontraram certa correspondência geral entre o comportamento dos formadores de políticas públicas e a opinião pública em certos tipos de questões. ${ }^{18}$ Uma segunda possibilidade, discutida na próxima seção, é que, em vez de afetar diretamente a formação de políticas, a opinião pública se constitui em um dos elementos das "condições de fundo", ou ambiente, em que o processo político se desdobra. ${ }^{19} \quad$ Uma terceira possibilidade, discutida mais adiante, é que a relação existente entre a opinião e as políticas não é de fato linear, mas dialética, no sentido de que não apenas a opinião afeta as políticas, mas também estas afetam aquela. ${ }^{20}$

\footnotetext{
${ }^{16}$ Os problemas com maior chance de serem afetados pela síndrome da atenção às questões, no dizer de Downs, são aqueles que compartilham três características básicas. Primeiro, são problemas que afetam adversamente uma minoria da população. Segundo, são problemas gerados como efeitos colaterais de arranjos que beneficiam uma maioria da população. Terceiro, são problemas capazes de gerar momentos "dramáticos", mas não períodos contínuos de atenção dos meios de comunicação. Em conjunto, essas características asseguram que: ... a maioria das pessoas não se lembrará continuamente do problema por seu próprio sofrimento; ... a solução do problema requer atenção e esforços continuados, além de mudanças fundamentais nas instituições e comportamentos sociais; ... (e) o foco sustentado dos meios de comunicação sobre o problema rapidamente aborrece a maioria do público. Downs, "Up and Down with Ecology, p. 42." Esta discussão deixa aberta a possibilidade de outros tipos de formulação de agenda que poderiam ocorrer quando um problema deixa de se enquadrar em alguma das três condições. Downs não se deteve nesses tipos de questões, porém, ou sobre os padrões típicos prováveis de desenvolvimento e mudanças de política que poderiam caracterizá-los.

${ }^{17}$ Hogwood, Brian W., Ups and Downs: Is There an Issue-Attention Cycle in Britain?, Glasgow, Strathclyde Papers in Government and Politics 89, 1992; Peters, B. Guy, e Brian W. Hogwood, In Search of the Issue-Attention Cycle, Journal of Politics 47 (1) 1985: 238-253; e, mais recentemente, Howlett, Michael, Issue-Attention and Punctuated Equilibria Models Reconsidered: Na Empirical Examination of the Dynamics of Agenda Setting in Canada, Canadian Journal of Political Science 30 (1) 1997.

${ }^{18}$ Ver, por exemplo, Weber, Ronald E., e William R. Shaffer, Public Opinion and American State Policy-Making, Midwest Journal of Political Science 16 1972: 683-699, e Bennett, W. Lance, Public Opinion in American Politics, New York, Harcourt Brace Jovanovich, 1980.

${ }^{19}$ Hoberg, George, Distinguishing Learning from Other Sources of Policy Change: The Case of Forestry in the Pacific Northwest, Boston: Trabalho apresentado na reunião anual da American Political Science Association, 1998.

${ }^{20}$ Ver Page, Benjamin I., e Robert Shapiro, Effects of Public Opinion on Policy, American Political Science Review 77 (1) 1983: 175-190, e Qualter, Terence H., Opinion Control in the Democracies, Londres, Macmillan, 1985.
} 


\section{Os Efeitos da Opinião Pública na Formação de Políticas (2): efeitos indiretos sobre os resultados como condição de fundo no ambiente das políticas}

Um modo de aproximar a questão da natureza indireta dos efeitos da opinião sobre as políticas é examinar mais detalhadamente o papel desempenhado pela opinião pública em cada etapa do processo de formação de políticas. Ao desagregar a formação de políticas em uma seqüência de etapas ou estágios discretos podemos discernir exatamente se e quando a opinião pública afeta a formação de políticas e onde tem menos significação que outros fatores. ${ }^{21}$

Um número razoavelmente limitado e consistente de etapas de políticas pode ser derivado se se conceber a formação de políticas como um processo de solução aplicada de problemas. Utilizando uma metáfora da solução de problemas, o processamento das políticas pode ser quebrado em etapas discretas em que um problema é identificado pelo governo, soluções são propostas, alguma solução é escolhida e depois implementada, e os resultados são monitorados para assegurar que o problema foi corrigido ou resolvido. Neste modelo, a construção da agenda se refere ao processo pelo qual os problemas chegam à atenção do governo; a formulação de políticas se refere ao processo pelo qual as opções por políticas são desenvolvidas dentro do governo; a tomada de decisões se refere ao processo pelo qual os governos adotam um curso particular de ação ou não ação; a implementação de políticas se refere ao processo pelo qual os governos põem as políticas em execução; e a avaliação de políticas se refere aos processos pelos quais os resultados das políticas são monitorados pelos atores estatais e societais, e cujo resultado pode ser a reformulação dos problemas e soluções. ${ }^{22}$

Este modelo dos ciclos das políticas facilita o entendimento da formação de políticas públicas quebrando a complexidade do processo em qualquer quantidade de etapas e sub-etapas, cada uma das quais pode ser estudada isoladamente, ou em termos

\footnotetext{
${ }^{21}$ A idéia de simplificar a complexidade da formação de políticas públicas quebrando o processo de formação de políticas em um número de etapas discretas foi mencionada por primeira vez nos primeiros trabalhos dos fundadores das "ciências da política". No trabalho do teórico da Universidade de Chicago Harold Lasswell, por exemplo, o processo político começava com a coleta de inteligência, isto é, a coleta, processamento e disseminação de informações para os participantes na tomada de decisões. Passava então à promoção de opções particulares antes que os tomadores de decisão prescrevessem um curso de ação. No próximo estágio, o curso de ação prescrito era invocado; o que quer dizer que era desenvolvido um conjunto de sanções para penalizar os que deixassem de seguir as prescrições dos tomadores de decisão. A política era então aplicada pelas cortes e pela burocracia e seguia seu caminho até terminar ou ser cancelada. Finalmente, os resultados da política eram apreciados e avaliados em relação aos alvos e objetivos dos tomadores da decisão original. Ver Harold Lasswell, The Decision Process: Seven Categories of Functional Analysis, College Park, University of Maryland, 1956, e Harold Lasswell, A Pre-View of Policy Sciences, New York, American Elsevier, 1971. Esses trabalhos antigos formam a base para modelos posteriores, como aqueles formulados por Gary Brewer no início dos anos 70. De acordo com Brewer, o processo das políticas era composto de seis etapas: Invenção/Início, Estimativa, Seleção, Implementação, Avaliação e Fim. Garry D. Brewer, The Policy Sciences Emerge: To Nurture and Structure a Discipline, Policy Sciences 5 1974: 239-244. O modelo foi popularizado na década de 80, ver Charles O Jones, Na Introduction to the Study of Public Policy, Monterrey, California, Brooks/Cole, 1984; James E. Anderson, Public Policy Making, New York, Praeger, 1984, 3a. edição, e Garry Brewer e Peter DeLeon, The Foundations of Policy Analysis, Homewood, Dorsey, 1983.

${ }^{22}$ Ver Michael Howlett e M. Ramesh, Studying Public Policy: Policy Cycles and Policy Subsystems, Toronto, Oxford University Press, 1995.
} 
de sua relação com qualquer uma ou com todas as outras etapas do ciclo. Isso auxilia a construção de teorias, ao permitir muitos estudos de caso e também estudos comparativos de diferentes etapas. Permite também o exame do papel de vários atores e instituições no processo - inclusive da opinião pública. ${ }^{23}$ No próximo item será discutido e avaliado o papel da opinião pública em cada etapa do processamento das políticas.

\section{A Opinião Pública e a Construção da Agenda}

O problema central para o entendimento do papel da opinião pública no processo de construção da agenda é compreender como surgem as demandas por uma política, como elas são articuladas por atores específicos e como emergem na agenda do governo. $^{24}$ Embora freqüentemente se suponha que esse processo começa com a expressão pública de alguma preocupação com alguma política, isso nem sempre acontece. Em muitos casos, de fato, atores estatais tomam a frente no processo. A agenda oficial do governo é usualmente dominada por oportunidades rotineiras ou institucionalizadas de construção da agenda mais que por aquelas geradas por eleições ou outras formas de atividade política partidária. ${ }^{25}$

Diversos atores políticos representam o público no processo de formação de políticas. No sentido mais geral, o público é tratado como uma entidade amorfa e passiva, cujo pulso é medido por pesquisas atitudinais. Tipicamente, o público difuso serve apenas como barômetro no processo político, e apenas grupos organizados do público fazem pressão por certos objetivos políticos. ${ }^{26}$

O melhor barômetro da opinião pública é obviamente a pesquisa de opinião pública. Mas ela não é, em si mesma, um ator no processo. As pesquisas geram informação que pode ser utilizada por outros atores, sejam eles representantes do governo, de interesses sociais ou outros. Esse nível baixo de envolvimento público na construção da agenda, porém, pode subir em campanhas de redação de cartas e petições, formação de grupos de trabalho sobre questões específicas, mobilização de

\footnotetext{
${ }^{23}$ A principal desvantagem desse modelo é que ele pode ser mal interpretado como se sugerisse que os formuladores de políticas se dedicam a resolver os problemas públicos de maneira muito sistemática e linear. Para uma avaliação crítica do modelo dos ciclos de políticas ver Hank C. Jenkins-Smith e Paul A. Sabatier, The Study of the Public Policy Processes, in Paul A. Sabatier e Hank C. Jenkins-Smith (orgs), Policy Change and Learning: Na Advocacy Coalition Approach, Bouder, Westview, 1993: 1-9. Para uma defesa do modelo, apesar de suas limitações, ver DeLeon, Peter, The Stages Approach to the Policy Process: What Has it Done? Where Is it Going?, in P. A. Sabatier (org) Theories of the Policy Process, Boulder, Westview, 1999: 19-34.

${ }^{24}$ Cobb, Roger W., e Charles D. Elder, Participation in American Politics: The Dynamics of Agenda Building, Boston, Allyn and Bacon, 1972. Em termos mais gerais, ver Malcolm Spector e John I. Kitsuse, Constructing Social Problems, New York, Aldine de Gruyter, 1987: 75-76.

${ }^{25}$ Ver Howlett, Michaes, Predictable and Unpredictable Policy Windows: Issue, Institutional and Exogenous Correlates of Canadian Federal Agenda-Setting, Canadian Journal of Political Science 31 (3) 1998. Ver também John W. Kingdon, Agendas, Alternatives and Public Policies, Boston, Little, Brown and Company, 1984, e Jack L. Walker, Setting the Agenda in the U.S. Senate: A Theory of Problem Selection, British Journal of Political Science 7 1977: 423-445.

${ }^{26}$ Pross, Paul, Group Politics and Public Policy, Toronto, Oxford University Press, 1992.
} 
piquetes e protestos e outras formas de "desobediência" civil pacífica ou violenta. ${ }^{27}$ Cidadãos individuais também podem expressar ao governo suas preocupações e opiniões através de vários procedimentos que facilitam e complementam os processos políticos estabelecidos. Esses procedimentos vão desde a pressão sobre representantes eleitos até a participação em corpos consultivos ou conselhos especializados. ${ }^{28}$

Todos esses tipos de ação pública podem ter efeito significativo na formação de políticas em áreas geográficas ou em questões específicas. Mas embora os formuladores de políticas estejam em geral interessados nas percepções difusas do público mais amplo, e devam levar a sério os atos de desobediência civil, essas expressões do interesse público tendem a ser pontuais, ad hoc e idiossincráticas. O que não quer dizer que sejam ineficazes, porque freqüentemente capturam a atenção dos meios de comunicação e podem ser centrais para atrair a atenção política para uma questão.

Os meios de comunicação desempenham um papel muito ativo e continuado, influenciando e refletindo a construção da agenda. Influencia a formação de políticas ao gerar a atenção do público e, através dela, a pressão política para que certos atores passem a atuar sobre uma questão particular. $^{29}$ A cobertura da mídia, porém, não apenas aumenta as percepções e atenção públicas sobre várias questões, mas as constrói, definindo-as como econômicas ou políticas, sociais ou pessoais, radicais ou conservadoras. O que é relatado, como é relatado, quem relata e o caráter do meio de comunicação, tudo isso tem implicações para a mensagem da mídia ao público. ${ }^{30}$ No entanto, embora a opinião pública faça o pano de fundo sobre o qual a mídia atua, existem várias limitações ao papel da mídia como ator no processamento das políticas que a tornam um agente mediador imperfeito para a opinião pública e faz com que a ligação entre a opinião pública e a formação de políticas seja indireta. Muitas questões crônicas permanecem sem ser noticiadas ou desenvolvidas pela mídia, por exemplo. A entrada de uma questão na agenda da mídia não é automática e os vieses da mídia resultam em níveis inadequados ou flutuantes de cobertura de muitas questões de grande saliência pública e, portanto, em uma apresentação não apropriada de questões aos governos. $^{31}$

\footnotetext{
${ }^{27}$ Riedel, James A., Citizen Participation: Myths and Realities, Public Administration Review, maio-junho 1972: 211-220.

${ }^{28}$ Ver Graham, Katherine A., e Susan D. Phillips, Citizen Engagement: Beyond the Customer Revolution, Canadian Public Administration 40 (2) 1997: 225-273, e Dion, Leon, The Politics of Consultation, Government and Opposition 8 (3) 1973: 332-353.

${ }^{29}$ Gibson, Robert, Out of Control and Beyond Understanding: Acid Rain as a Political Dilemma, in Paehlke, Robert, e Douglas Torgerson (orgs), Environmental Politics and the Administrative State, Peterborough, Broadview Press, 1990: 248.

${ }^{30}$ Cook, F. L. e outros, Media and Agenda-Setting: Effects on the Public, Interest Group Leaders, Policy Makers and Policy, Public Opinion Quarterly 47 (1) 1983: 16-35.

${ }^{31}$ Erbring, Lutz, e Edie N. Goldenberg, Front Page News and Real World Cues: A New Look at Agenda-Setting by the Media, American Journal of Political Science 24 (1) 1980: 16-49; McCoombs, Maxwell E., The Agenda-Setting Approach, in Handbook of Political Communication, orgs. D. D. Nimmo e K. R. Sanders, Beverly Hills, Sage, 1981: 121-140. Os jornalistas, é claro, também encontram outras limitações, na forma de recursos, tempo e espaço inadequados, falta de apoio superior, e as práticas tradicionais e interesses econômicos de seus empregadores.
} 
Por essas e por outras razões, muitos observadores do comportamento na construção da agenda nas sociedades democráticas contemporâneas concluíram que o principal impacto da opinião pública nesta etapa da formação de políticas é sentido antes no estilo de construção da agenda adotado em certos setores ou jurisdições do que em seu conteúdo. Como estudiosos desde Cobb, Ross e Ross até Peter May assinalaram, a existência ou não de um público em determinada questão pode afetar e de fato afeta as atividades de construção da agenda através da definição dos tipos de processo de construção de agenda seguidos pelo governo. ${ }^{32}$ Em questões com grande público, os governos podem ser pressionados a agir, enquanto que naquelas com pouco público é mais difícil aumentar a pressão. De modo semelhante, em questões com grande público, as iniciativas do governo devem ser legitimadas por processos de consulta e audiências públicas, ao passo que, sem público, as questões podem ser tratadas internamente. Nos dois casos, porém, isso significa que o conteúdo real da opinião pública é menos significativo do que o simples fato de que a questão envolva um grande ou pequeno segmento do público.

\section{A Opinião Pública e a Formulação de Políticas}

A opinião pública tem ainda menos significação direta para a formulação de políticas públicas ou para a proposta de opções para lidar com problemas políticos. Isto é, o público não é, em geral, representado diretamente nos sub-sistemas das políticas setoriais que desenvolvem tais opções. ${ }^{33} \mathrm{Na}$ melhor das hipóteses, ele poderá ter representação indireta através de grupos de interesse, representantes eleitos, administradores governamentais ou outros membros dessas organizações centrais.

O pertencimento e a dinâmica do subsistema de políticas desde há muito atrai a atenção dos estudiosos da formulação de políticas públicas. Identificar quais são os atores-chave, o que os aproxima, como interagem e que efeitos sua interação tem sobre as políticas são os principais objetivos nessa tradição. Os pesquisadores têm desenvolvido vários modelos para dar conta dessas questões. ${ }^{34} \quad$ Uma maneira útil de

\footnotetext{
${ }^{32}$ Ver Cobb, R., J. K. Ross e M. H. Ross, Agenda Building as a Comparative Political Process, American Political Science Review 70 (1) 1976: 126-138, e May, Peter J., Reconsidering Policy Design: Policies and Publics, Journal of Public Policy 11 (2) 1991: 187-206.

${ }^{33}$ Ver Michael Howlett e M. Ramesh, Studying Public Policy: Policy Cycles and Policy Subsystems, Toronto, Oxford University Press, 1995; Rhodes, R. A. W., Understanding Governance: Policy Networks, Governance, Rfexivity and Accountability, Buckingham, Open University Press, 1997; Lembruch, Gerhard, The Organization of Society, Administrative Strategies, and Policy Networks, in R. M. Czada e A. Windhoff-Heritier (orgs) Political Choice: Institutions, Rules, and the Limits of Rationality, Boulder, Westview, 1991: 121-155; Knoke, David, Networks as Political Glue: Explaining Public Policy-Making, in W. J. Wilson (org) Sociology and the Public Agenda, Londres, Sage, 1993: 164184

${ }^{34}$ Grant Jordan discutiu as muitas imagens e metáforas utilizadas para descrever os subsistemas de políticas. Ver Grant Jordan, Iron Triangles, Woolly Corporatism and Elastic Nets: Images of the Policy Process, Journal of Public Policy 1 1981: 95-123; Grant Jordan, Policy Community Realism versus 'New' Institutionalist Ambiguity, Political Studies 38 1990: 470-484; Grant Jordan, Subgovernmanets, Policy Communities and Networks: Refilling the Old Bottles?, Journal of Theoretical Politics 2 1990: 319-338; Grant Jordan e Klaus Schubert, A preliminary Ordering of Policy Network Labels, European Journal of Political Research 21 1992: 7-27.
} 
pensar nesse problema é relacioná-lo à idéia de vários subsistemas que operam dentro do sistema político maior, processando questões de políticas de forma paralela a fim de aliviar o peso de informações e decisões que é colocado sobre as instituições centrais do governo democrático. ${ }^{35}$ As realidades dos papéis desempenhados pelo poder político e pelo conhecimento técnico especializado no processamento das políticas criam limitações significativas para os membros do subsistema e servem para restringir severamente o impacto da opinião pública sobre atividades que se desenvolvem nesta etapa. Uma limitação organizacional significativa da opinião pública está relacionada à natureza técnica de muitas questões políticas, o que vem a limitar o papel que poderia ser desempenhado por um público com uma formação generalista. Isto é, membros do público geralmente não compartilham a formação científica e a educação técnica especializada da maioria dos burocratas e funcionários. A participação na formulação de políticas requer que os membros do público de uma questão sejam capazes de, no mínimo, construir a questão como problemática, e tenham informação suficiente para buscar soluções potenciais para o problema. Em muitos casos, só os especialistas têm essa capacidade.

Isso significa que o que é importante no estágio da formulação de políticas não é tanto a opinião pública, ou os mecanismos para a apresentação direta da opinião pública aos formuladores de políticas como os meios de comunicação, mas a existência de instituições e organizações públicas quase-representativas - como grupos de interesse e partidos políticos - que podem reunir o conhecimento relevante requerido para participar da formulação de políticas. ${ }^{36}$ Esses grupos, com seus correspondentes estatais, formam a espinha dorsal dos subsistemas de políticas. ${ }^{37}$

No entanto, ainda aqui existem problemas significativos com a organização da apresentação mesmo indireta da opinião pública aos formuladores das políticas através desses grupos intermediários. Isto é, os grupos de interesse encontram contínuos problemas em atingir e reter os altos níveis de participação requeridos para o financiamento necessário para manter suas atividades e legitimar sua capacidade de falar

\footnotetext{
${ }^{35}$ Jones, Bryan D., Reconceiving Decision-Making in Democratic Politics: Attention, Choice and Public Policy, Chicago, University of Chicago Press, 1994.

${ }^{36}$ Paul Sabatier, Knowledge, Policy-Oriented Learning, and Policy Change, Knowledge: Creation, Diffusion, Utilization 8 1987: 664

37 A mais antiga concepção de um subsistema de políticas envolvia a noção de um 'sub-governo', consistindo de agrupamentos de atores societais e estatais em padrões rotinizados de interação. Lance deHaven-Smith e Carl E. Van Horn, Subgovernment Conflict in Public Policy, Policy Studies Journal 12 1984: 627-642. O conceito foi desenvolvido nos Estados Unidos nos anos 50 na base da observação de que os grupos de interesse, comitês do congresso e agencias governamentais naquele país desenvolviam um sistema de apoio mútuo no curso de uma constante interação sobre questões legislativas e regulatórias. Essas relações triangulares em áreas como agricultura, transportes e educação foram às vezes denominadas de 'triângulo de ferro' a fim de expressar a essência de seu férreo controle sobre muitos aspectos do processamento das políticas. Douglas Cater, Power in Washington: A Critical Look at Today's Struggle in the Nation's Capital, New York, Random House, 1964. Pesquisa subsequente do caso norte-americano nos anos 60 e 70, porém, revelou que esses sub-governos não eram todo-poderosos, e que de fato sua influência sobre a formulação de políticas era variada, dependendo de questão e de momento. Logo surgiu uma noção menos rígida do subsistema de políticas, chamada de rede de questões. Hugh Heclo, Issue Networks and the Executive Establishment, in Anthony King (org), The New American Political System, Washington, D.C., American Enterprise Institute for Public Policy Research 1978: 87-124.
} 
em nome de um segmento da população. Isso ocorre porque, embora membros do público possam se beneficiar de sua representação através de um grupo de interesse, há pouca motivação material direta para sua contribuição para apoiar um grupo. Membros do público podem facilmente pegar 'carona', participando dos ganhos produzidos pela ação dos grupos de interesse sem pagar qualquer dos custos, e, portanto, enfraquecendo o funcionamento do sistema de articulação de interesses e de representação. ${ }^{38}$

Os partidos políticos também encontram dificuldades na transmissão da opinião pública ao governo. Como veículos para a eleição de líderes e governos, os partidos articulam plataformas políticas que contêm numerosas propostas de políticas. Como os grupos de interesse, também Ihes faltam recursos financeiros, bem como o conhecimento especializado que permitiriam que essas propostas pudessem competir com aquelas desenvolvidas pelos funcionários especializados e permanentes. ${ }^{39}$ Como resultado, a maioria das prescrições políticas partidárias são ignoradas pelos próprios membros dos partidos quando eleitos. ${ }^{40}$

\section{A Opinião Pública e a Tomada de Decisões}

Quase por definição, o número de atores políticos relevantes diminui à medida em que a questão caminha através das várias etapas do processamento das políticas públicas. Dessa forma, a construção da agenda pode envolver uma ampla variedade de atores estatais e societais, enquanto que no estágio da formulação de políticas, o número de atores permanece grande, mas é reduzido para incluir apenas aqueles que formam algum subsistema de política setorial. Com a exceção dos relativamente raros referendos e plebiscitos, o estágio de tomada de decisões em políticas públicas envolve ainda menos atores, normalmente excluindo quase todos os atores não-estatais, assim como os que pertencem a outros ramos do governo. Apenas os políticos, juízes e funcionários autorizados a tomar decisões na área em questão participam desse estágio do ciclo da política. Isso confere aos líderes políticos extensa influência sobre os resultados das políticas..$^{41}$

Em governos modernos, porém, o grau de liberdade desfrutado pelos que tomam decisões é circunscrito a um conjunto de regras que governam cada agência e limitam os seus responsáveis. Essas regras vão desde a constituição do país até os mandatos

\footnotetext{
${ }^{38}$ Olson, Mancur, The Logic of Collective Action: Public Goods and the Theory of Groups, Cambridge, Harvard University Press, 1965.

39 Joel D. Aberbach, Robert D. Putnam e Bert A. Rockman, Bureaucrats and Politicians in Western Democracies, Cambridge, Harvard University Press, 1981.

${ }^{40}$ D. A. Hibbs Jr., Partisan Theory After Fifteen Years, European Journal of Political Economy 8 1992: 361-373; F. G. Castles e R. D. McKinlay, Reflections: Does Politics Matter? Increasing Complexity and Renewed Challenges, European Journal of Political Research 31 (1-2) 1997: 102-107; Manfred G. Schmidt, When Parties Matter: A Review of the Possibilities and Limits of Partisan Influences on Public Policy, European Journal of Political Research 30 1996: $155-183$. ${ }^{41}$ Ver Leslie Pal, Hands at the Helm? Leadership and Public Policy, in Leslie Pal e David Taras (orgs), Prime Ministers and Premiers: Political Leadership and Public Policy in Canada, Scarborough, Prentice-Hall, 1988: 16-26, e Thomas S. Axworthy, Of Secretaries to Princes, Canadian Public Administration 31 (2) 1988.
} 
específicos conferidos aos indivíduos que tomam decisões através de diversas leis e regulamentos. As regras usualmente definem não só as decisões que podem ser tomadas pelas agências ou funcionários do governo, mas também os procedimentos que devem ser seguidos. Diferentes países têm diferentes arranjos constitucionais e diferentes conjuntos de regras governando a estrutura de agências governamentais e a conduta dos funcionários. Como observaram Allison e Halperin, tais regras de procedimento fornecem 'canais de ação' aos tomadores de decisões, isto é, um conjunto regularizado de procedimentos para produzir certos tipos padrão de decisões. ${ }^{42}$ Embora o propósito das regras seja circunscrever a liberdade dos tomadores de decisões e forçálos a registrar suas deliberações de modo a permitir avaliação subseqüente, uma considerável autonomia permanece com os indivíduos para chegar ao seu próprio juízo sobre 'o melhor' curso de ação a seguir em circunstâncias específicas.

Exatamente qual o processo seguido e qual a decisão considerada 'a melhor' varia entre tomadores de decisões e nos contextos em que operam. Os tomadores de decisão variam muito em termos de sua formação, conhecimento e predileções, o que afeta o modo como interpretam um problema e suas soluções. ${ }^{43}$ Diferentes tomadores de decisão operando em situações institucionais semelhantes respondem diferentemente ao lidar com os mesmos problemas ou com problemas semelhantes.

A despeito das circunstâncias específicas, porém, o executivo é isolado de muitas influências diretas, inclusive as da opinião pública. ${ }^{44}$ Isso não quer dizer que a opinião pública não influencia a tomada de decisões pelo executivo num nível mais geral. Ela pode afetar a tomada de decisões na medida em que os governos tentam posicionar-se contra seus rivais partidários na preparação de campanhas eleitorais futuras, ${ }^{45}$ ou pela criação de um 'clima de políticas' geral que os formadores de políticas podem refletir, ou do qual podem tomar conhecimento em suas deliberações. ${ }^{46}$

\footnotetext{
${ }^{42}$ Graham T. Allison e Morton H. Halperin, Bureaucratic Politics: A Paradigm and Some Policy Implications, World Politics 24 (Suplemento) 1972: 40-79.

${ }^{43}$ Ralph K. Huitt, Political Feasibility, in Austin Ranney (org) Political Science and Public Policy, Chicago, Markham Publishing Co., 1968.

${ }^{44}$ Richard Rose, The Problem of Party Government, New York, Free Press, 1974: 306. Sobre a situação geral em relação a divergências nos valores da 'elite' e da 'massa' que isso engendra, ver Paul M. Sniderman, Joseph F. Fletcher, Peter H. Russell, Phillip E. Tetlock e Brian J. Gaines, The Fallacy of Democratic Elitism: Elite Competition and Commitment to Civil Liberties, British Journal of Political Science 21 1991: 349-370.

${ }^{45}$ Essa é a atividade de 'contágio' identificada por Maurice Duverger em seu estudo dos partidos políticos na Europa. Para uma aplicação e exame empírico dessa tese no Canadá, ver W. M. Chandler, Canadian Socialism and Policy Impact: Contagion from the Left, Canadian Journal of Political Science 10 (4) 1977: 755-780, e François Petry, The Policy Impact of Canadian Party Programs: Public Expenditure Growth and Contagion from the Left, Canadian Public Policy 14 (4) 1988: 376-389.

${ }^{46}$ Stimson, James A., Public Opinion in America: Moods Cycles and Swings, Boulder, Westview, 1991; Durr, Robert H., What Moves Policy Sentiment, American Political Science Review 87 1993: 158-172; Best, Samuel J., The Sampling Problem in Measuring Policy Mood: An Alternative Solution, The Journal of Politics 61 (3) 1999: 721-740.
} 


\section{A Opinião Pública e a Implementação de Políticas}

Funcionários indicados assessoram o executivo no desempenho de suas tarefas, como sugerido pelos termos 'servidores civis' ou 'servidores públicos'. No entanto, a realidade do governo moderno é tal que seu papel vai muito além do que se esperaria de um 'servidor'. De fato, os burocratas são freqüentemente os atores centrais em processos políticos e as figuras centrais em muitos subsistemas de políticas. ${ }^{47} \quad \mathrm{O}$ tremendo crescimento em tamanho, alcance e complexidade das funções de governo ao longo dos anos impede que políticos generalistas controlem, ou até mesmo percebam, as atividades específicas de governo nominalmente sob seu controle. ${ }^{48}$ A maioria das funções de implementação antigamente desempenhadas por legislaturas e pelo executivo político são hoje desempenhadas pela burocracia. Muitas funções do governo moderno são complexas e numerosas demais para serem desempenhadas por algumas dezenas de membros do gabinete, enquanto que a burocracia consiste de grande número de especialistas que têm o tempo e a especialização necessários para lidar com a questão política em base contínua.

O poder e a influência da burocracia sobre a implementação de políticas baseiase na amplitude dos recursos politicamente relevantes que ela controla. ${ }^{49}$ A própria lei freqüentemente determina que certas funções sejam desempenhadas pela burocracia. Isso freqüentemente confere ampla autonomia a burocratas individuais para tomar decisões pelo estado. As burocracias têm também amplo acesso a recursos materiais para buscar seus próprios objetivos organizacionais e mesmo pessoais, se assim quiserem. O governo é a maior unidade de despesas na maioria dos países, se não em todos, situação que dá a seus funcionários uma voz poderosa nas áreas de políticas que envolvem gastos governamentais significativos. A burocracia é também um repositório de ampla gama de habilidades e especialidades, recursos que fazem dela uma organização importante na sociedade. Ela emprega grande número de quase todos os tipos de profissionais que são empregados devido a seu status como especialistas em determinadas áreas. $O$ fato de que tratam de questões semelhantes em base continuada dota-os de percepções únicas dos problemas. Além disso, as modernas burocracias têm acesso a vastas quantidades de informação sobre muitos aspectos diferentes da sociedade. Em certos momentos, a informação é coletada deliberadamente, mas em outros a informação flui para ela como parte de seu lugar central no governo. A

\footnotetext{
${ }^{47}$ R. F. Adie e P. G. Thomas, Canadian Public Administration: Probematical Perspectives, Scarborough, Prentice-Hall, 1987; K. Kernaghan, Power, Parliament and Public Servants in Canada: Ministerial Responsibility Reexamined, Canadian Public Policy 5 1979: 383-396; K. Kernaghan, The Public and Public Servant in Canada, in K. Kernaghan (org), Public Administration in Canada: Selected Readings, Toronto, Methuen, 1985: 323-330.

${ }^{48}$ John Markoff, Governmental Bureaucratization: Genral Processes and na Anomalous Case, Comparative Studies in Society and History 17 (4) 1975: 479-503; Edward C. Page, Political Authority and Bureaucratic Power: A Comparative AnalysisBrighton, Sussex, Wheatsheaf, 1985.

${ }^{49}$ Larry B. Hill, Introduction, in Larry B. Hill (org), The State of Public Bureaucracy, Armonk, N.Y., M. E. Sharpe, 1992: $1-11$.
} 
permanência da burocracia e a estabilidade de seus membros também Ihe dão uma vantagem sobre seus superiores, o executivo eleito. Finalmente, o fato de que as deliberações sobre políticas em sua maior parte ocorrem em segredo dentro da burocracia nega a outros atores a oportunidade de preparar uma oposição a seus planos.

A burocracia é altamente isolada da pressão pública devido a várias características estruturais e pessoais que servem para proteger os servidores públicos do escrutínio público ou de penalidades. ${ }^{50}$ Esse isolamento, porém, não deve ser exagerado. O executivo é responsável em última instância por todas as políticas, autoridade que ele afirma algumas vezes. A implementação de grandes questões políticas tende a envolver níveis mais altos de controle executivo. $\mathrm{O}$ controle pelo executivo tende também a ser maior se a burocracia consistentemente se opõe às opções políticas preferidas pelos políticos ou pelo público. Mais ainda, a própria burocracia não é uma organização homogênea, mas antes uma coleção de organizações, cada uma com seus próprios interesses, perspectivas e procedimentos padronizados de operação. Mesmo dentro do mesmo departamento, há muitas vezes divisões que seguem as linhas funcional, pessoal, política e técnica. Não é incomum para o executivo intervir para resolver conflitos intra e interburocráticos. ${ }^{51}$ De qualquer maneira, há poucas dúvidas de que esta etapa do processamento de políticas é muito pouco afetado pela opinião pública, a menos que os administradores exijam que suas ações sejam consideradas legítimas a fim de assegurar o cumprimento das regras e regulamentos governamentais. ${ }^{52}$

\section{A Opinião Pública e a Avaliação de Políticas}

O ato de votar é muitas vezes concebido em termos de avaliação de políticas, ou seja, como uma atividade coletiva em que eleitores individuais julgam as políticas de um governo. No entanto, embora o ato de votar seja o mais básico e importante meio de participar dos processos políticos dos estados democráticos, ele dá aos eleitores a oportunidade de expressar apenas sua escolha geral de governo, mais que sua escolha de políticas específicas. ${ }^{53} \mathrm{Em}$ outras palavras, ainda que os partidos e os candidatos em busca de votos possam tentar oferecer pacotes de políticas que, esperam, atrairão os

\footnotetext{
50 Aucoin, Peter, The Design of Public Organizations for the $21^{\text {st }}$ Century: Why Bureaucracy Will Survive in Public Management, Canadian Public Administration 40 (2) 1997: 290-306; Egeberg, Morten, The Impact of Bureaucratic Structure on Policy Making, Public Administration 77 (1) 1999: 155-170.

${ }^{51}$ Sharon L. Sutherland, The Public Service and Policy Development, in M. Michael Atkinson (org), Governing Canada: Institutions and Public Policy, Toronto, Harcourt Brace Jovanovich, 1993.

52 Beetham, David, The Legitimation of Power, Londres, Macmillan, 1991; Habermas, Jurgen, Legitimation Crisis, Boston, Beacon Press, 1975; Mayntz, Renate, Legitimacy and the Directive Capacity of the Political System, in L. N. Lindberg et alii (orgs) Stress and Contradiction in Modern Capitalism, Lexington, Lexington Books, 1975: $261-274$. Ver também Hawkins, Keith, e John M. Thomas, Making Policy in Regulatory Bureaucracies, in J. M. Thomas (org), Making Regulatory Policy, Pittsburgh, University of Pittsburgh Press, 1989: 3-30.

${ }^{53}$ George C. Edwards III e Ira Sharkansky, The Policy Predicament: Making and Implementing Public Policy, San Francisco, Freeman, 1978: 23.
} 
eleitores, o sistema eleitoral não é estruturado de modo a permitir aos eleitores uma escolha de políticas específicas. ${ }^{54}$

De fato, a maioria das avaliações é realizada por funcionários ou membros do judiciário, que são isolados da opinião e da pressão públicas. ${ }^{55}$ Provavelmente as formas de avaliação mais próximas do público são os diferentes tipos de revisões legislativas. Mesmo aqui, entretanto, o efeito da opinião pública sobre a formação de políticas é fraco e muito indireto. Em muitas legislaturas contemporâneas, as funções de avaliação de políticas não são desempenhadas no plenário mas nas comissões estabelecidas com base em bancadas funcionais para analisar a legislação proposta. As comissões freqüentemente atingem competência considerável na área de sua atuação, e essa competência permite que o legislativo exerça sua influência sobre o processamento das políticas. Mas para construir essa competência, os membros devem participar das comissões por períodos relativamente longos de tempo. Os membros das comissões também não devem seguir necessariamente a posição de suas bancadas ao votar, a fim de manter sua autonomia. ${ }^{56}$ A natureza do problema considerado também afeta 0 envolvimento legislativo na avaliação de políticas. Questões técnicas e puramente legais dificilmente envolverão os legisladores, seja porque podem não compreender inteiramente os problemas ou soluções, seja porque podem ver benefícios políticos insuficientes na questão. Além disso, algumas áreas de políticas, como a segurança nacional e a política externa são usualmente tratadas numa teia de segredos, e fora do legislativo. De modo similar, políticas que dizem respeito a um problema percebido como crítico dificilmente envolvem muito o legislativo, por causa do tempo que toma a revisão de uma política. Tudo isso enfraquece as ligações que poderiam existir entre a opinião pública e a avaliação de políticas.

\footnotetext{
${ }^{54}$ Anthony King, What Do Elections Decide?, in David Butler, Howard R. Penniman e Austin Ranney (orgs), Democracy at the Polls: A Comparative Study of Competitive National Elections, Washington, D. C., American Enterprise Institute for Public Policy Research, 1981.

${ }^{55}$ Ver Jaffe, Louis, Judicial Control of Administrative Action, Boston, Little Brown, 1965.

${ }^{56}$ Ver David M. Olson e Michael L. Mezey, Parliaments and Public Policy, in David M. Olson e Michael Mezey (orgs) Legislatures in the Policy Process: The Dilemmas of Economic Policy, Cambridge, Cambridge University Press, 1991: 124.
} 


\section{A Dialética Política-Opinião: efeitos da formação de políticas sobre a opinião pública}

Como mostrou a discussão acima, ainda que a idéia de que a opinião pública determine os resultados de políticas diretamente seja insustentável, isso não quer dizer que a opinião pública não desempenhe um papel na formação de políticas em estados democráticos. Como mostrou a discussão do papel da opinião pública em cada estágio do processamento de políticas, a opinião pública desempenha importante papel indireto na formação de políticas por constituir elemento muito significativo do ambiente em que operam os sistemas e subsistemas de políticas. Ela afeta a natureza da construção da agenda mais fortemente do que qualquer dos outros estágios, mas tem também impacto significativo sobre a tomada de decisões porque forma parte importante do ambiente em que se desenvolve a formação de políticas. Esse papel é provavelmente menos visível na formulação, na implementação e na avaliação de políticas, embora, mesmo nesses casos, a necessidade de que as políticas encontrem um nível pelo menos nominal de apoio público assegure que ela terá algum impacto sobre as atividades dos atores políticos mais diretamente relevantes.

Toda a discussão até aqui enfocou apenas um dos lados da dialética políticasopinião pública: a idéia de que a relação existente entre opinião pública e a formação de políticas públicas, seja ela direta ou indireta, é uma relação em que a opinião pública leva a políticas. Mas, como dizem Margolis e Mauser, a opinião pública não é só uma variável independente; pode ser também uma variável dependente. ${ }^{57}$ Isto é, embora seja evidente que a opinião constitui importante aspecto do ambiente político que afeta as ações do governo, também essas ações podem afetar o ambiente político. Ou seja, os governos não são apenas recipientes e reagentes passivos da opinião pública, mas podem ter, e freqüentemente têm, um papel ativo na conformação da opinião pública. ${ }^{58}$ Este é um aspecto muitas vezes despercebido da relação entre políticas públicas e opinião pública, e muitas vezes apenas entra no sentido negativo de que a propaganda e outras atividades do governo servem para enfraquecer uma democracia que funciona. ${ }^{59}$

Existem, no entanto, numerosos instrumentos de políticas utilizados pelos governos que podem afetar a natureza e o nível de apoio público que esses governos, ou iniciativas políticas que tomam, desfrutam. Um óbvio exemplo da dialética opinião-

\footnotetext{
${ }^{57}$ M. Margolis e G. A. Mauser (orgs), Manipulating Public Opinion: Essays on Public Opinion as a Dependent Variable, Pacific Grove, Brooks/Cole, 1989.

58 Edelman, Murray J., Constructing the Political Spectacle, Chicago, University of Chicago Press, 1988; Bosso, Christopher J., Setting the Agenda: Mass Media and the Discovery of Famine in Ethiopia, in Manipulating Public Opinion: Essays on Public Opinion as a Dependent Variable, orgs. M. Margolis e G.A. Mauser, Pacific Grove: Brooks/Cole, 1989: 153-174.

${ }^{59}$ Ver Hughes, John E., e M. Margaret Conway, Public Opinion and Political Participation, in B. Norrander e C. Wilcox (orgs), Understanding Public Opinion, Washington, D.C., CQ Press, 1997: 191-210; Page, Benjamin I., e Robert Y. Shapiro, Effects of Public Opinion on Policy, American Political Science Review 77 (1) 1983: 175-190; Page, Benjamin I., e Robert Y. Shapiro, The Rational Public: Fifty Years of Trends in American Policy Preferences, Chicago, The University of Chicago Press, 1992.
} 
políticas diz respeito ao uso de instrumentos substantivos de políticas baseados em informação, como a propaganda e a educação ou campanhas de conscientização para atingir objetivos governamentais específicos. Os esforços do governo para alterar a opinião e o comportamento do público em questões como beber e dirigir, os riscos das drogas ou do cigarro para a saúde, a necessidade do respeito aos direitos humanos, ou mesmo a promoção de sentimentos de solidariedade nacional ou unidade nacional são conhecidos e documentados nos estudos sobre o tema. ${ }^{60}$

Mais recentemente, muitos governos têm também desenvolvido um renovado interesse em tipos de instrumentos de políticas que podem lidar com as complexidades do gerenciamento da opinião pública. ${ }^{61}$ Ou seja, os governos de muito países complementam hoje regularmente o uso de um número relativamente reduzido de ferramentas 'substantivas' tradicionais de política, mais ou menos na linha do comando e controle - tais como empresas públicas, agencias reguladoras, subsídios e incentivos que tentam influenciar diretamente a alocação de bens e serviços na sociedade, com o uso de um conjunto de ferramentas inteiramente diferente: instrumentos 'procedimentais' como parcerias entre governo e ONGs, comissões assessoras públicas, apoio a grupos de interesse e disseminação de informações que agem de modo menos direto a fim de guiar os atores sociais na direção que o governo deseja. ${ }^{62}$

\footnotetext{
${ }^{60}$ Salmon, Charles (org), Information Capaigns: Managing the Process of Social Change, Newberry Park, Sage, 1989; Weiss, Janet A., e Mary Tschirhart, Public Information Campaigns as Policy Instruments, Journal of Policy Analysis and Management 13 (1) 1994: 82-119; Adler, Robert S., e R. David Pittle, Cajolry or Command: Are Education Campaigns na Adequate Substitute for Regulation, Yale Journal on Regulation 1 1984: 159-193; McDonnell, Lorraine M., e Richard F. Elmore, Alternative Policy Instruments, Santa Monica, Center for Policy Research in Education, 1987.

${ }^{61}$ As ciências de políticas sempre estiveram interessadas no processamento de políticas e na maneira como os governos manipulam esses processos. Em seus trabalhos pioneiros sobre a formação de políticas públicas, por exemplo, Harold Lasswell considerava que os principais instrumentos políticos envolviam, entre outras coisas, a manipulação de símbolos, signos e ícones. Lasswell observou em que medida os governos podiam afetar cada etapa do processo político com tal manipulação e propôs que uma tarefa importante dessas ciências deveria ser a compreensão das nuanças dessas ações e de seus efeitos. Ver Harold Lasswell, Key Symbols, Signs and Icons, in Lymon Brison, Louis Finkelstein, R. M. MacIver e Richard McKean (orgs), Symbols and Values: Na Initial Study, New York, 1954; H. D. Lasswell, The Decision Process: Seven Categories of Functional Analysis, College Park, University of Maryland, 1956; e H. D. Lasswell, The Policy Orientation, in D. Lerner e H. D. Lasswell (orgs) The Policy Sciences: Recent Developments in Scope and Method, Stanford, Stanford University Press, 1951: 3-15. Para discussões mais recentes, ver Peters, B. Guy, Managing Horizontal Government: The Politics of Coordination, Public Administration 76 1998: 295-311; Peters, B. Guy, Managing Horizontal Governmanet: The Politics of Coordination, Ottawa, canadian Centre for Management Development, 1998; e Klijn, E. H., e G. R. Teisman, Effective Policy-Making in a Multi-Actor Setting: Networks and Steering, in R. In'T Veld, L. Schaap, C. J. A. M. Termeer e M. J. W. Van Twist (orgs), Autopoiesis and Configuration Theory: New Approaches to Societal Steering, Dordrecht, Kluwer, 1991: 99-111.

${ }^{62}$ Sobre as ferramentas políticas substantivas 'tradicionais', ver Woodside, K., Policy Instruments and the Study of Public Policy, Canadian Journal of Political Science 19 (4) 1986: 775-793, e Lester M. Salamon (org) Beyond Privatization: The Tools of Government Action, Washington, D.C., Urban Institute, 1989. Sobre os instrumentos 'procedimentais', ber Michael Howlett, Legitimzacy and Governance: Re-Discovering Procedural Policy Instruments, Vancouver, trabalho apresentado à reunião anual da British Columbia Political Studies Association, 1996, e in't Veld, Roeland J., The Dynamics of Instruments, in B. G. Peters e F. K. M. Van Nispen (orgs) Public Policy Instruments: Evaluating the Tools of Public Administration, New York, Edward Elgar, 1998: 153-162.
} 
O trabalho de Bressers \& Klok, de Schneider \& Ingram, e de outros, ${ }^{63}$ identificou diversos instrumentos de política tipicamente procedimentais. Eles incluem educação, treinamento, criação de instituições, disseminação de informações, avaliações formais, audiências e reforma institucional. A pesquisa sobre as ferramentas e mecanismos utilizados nos projetos reguladores intergovernamentais também identificou diversos outros instrumentos tais como 'tratados' e ampla gama de 'acordos políticos' que podem afetar o reconhecimento das intenções governamentais pelos grupos-alvo e vice-versa. ${ }^{64}$ Outras pesquisas sobre o comportamento e atividades de grupos de interesse mostraram a existência de ferramentas relacionadas à criação e manipulação de grupos, inclusive o papel desempenhado por patronos do setor privado e do público no auxílio à formação e às atividades desses grupos. ${ }^{65}$ Ainda outras pesquisas sobre a formação contemporânea de políticas observaram a utilização de técnicas como os grupos focais; ${ }^{66}$ financiamento de pesquisas para audiências e tribunais investigativos, inclusive o acesso a estes últimos. ${ }^{67}$

Finalmente, alguns pesquisadores também salientaram o modo como as ferramentas podem ser utilizadas para afetar negativamente o comportamento dos grupos de interesse e de outros atores. Tais instrumentos procedimentais 'negativos' incluem a supressão de informações, e o fingimento, ofuscação e outras formas de adiamento administrativo. ${ }^{68}$ Cada um desses instrumentos pode ser utilizado pelos governos para manipular a opinião pública e o apoio popular para suas políticas. Eles sublinham a noção de que a relação opinião-políticas não é linear, mas de natureza dialética. Ou seja, não só a opinião pode influenciar as políticas, mas também o contrário, e uma compreensão de ambas as relações é necessária a fim de entender propriamente os fenômenos.

\footnotetext{
${ }^{63}$ Ver, por exemplo, Janet A. Weiss e Mary Tschirhart, Public Information Campaigns as Policy Instruments, in Journal of Policy Analysis and Management 13 (1) 1994: 82-119; Robert Bellehumeur, Review: Na Instrument of Change, Optimum 27 (1) 1997: 37-42; e Michael Saward, Co-Optive Politics and State Legitimacy, Aldershot, Dartmouth, 1992.

${ }^{64}$ Ver Simon J. Bulmer, The Governance of the European Union: A New Institutionalist Approach, Journal of Public Policy 13 (4) 1993: 351-380.

${ }^{65}$ Sobre a situação da patronagem do setor privado nos EUA, ver Anthony Nownes e Grant Neeley, Toward na Explanation for Public Interest Group Formation and Proliferation: 'Seed Money', Disturbances, Entrepreneurship and Patronage, Policy Studies Journal 24 (1) 1996: 74-92. Sobre a situação da patronagem pública no Canadá, ver Leslie A. Pal, Interests of State: The Politics of Language, Multiculturalism and Feminism in Canada, Kingston, McGill-Queens University Press, 1993, e Sandra Burt, Canadian Women's Groups in the 1980s: Organizational Development and Policy Influence, Canadian Public Policy 16 1990: 17-28.

${ }^{66}$ Ver Scott R. Furlong, Reinventing Regulatory Development at the Environmental Protection Agency, Policy Studies Journal 23 (3) 1995: 466-482.

${ }^{67}$ Ver Jane Jenson, Commissioning Ideas: Representation and Royal Commissions, in Susan D. Phillips (org) How Ottawa Spends 1994-95: Making Change, Ottawa, Carleton University Press, 1994: 39-69, e Alan C. Cairns, Reflections on Commission Research, in A. Paul Pross, Innis Christie e John A. Yogis (orgs) Comissions of Inquiry, Toronto, Carswell, 1990: 87-110. Ver também Melody Hessing e Michael Howlett, Canadian Ressource and Environmental Policy: Political Economy and Public Policy, Vancouver, University of British Columbia Press, 1997; Salter, Liora, e Debra Slaco, Public Inquiries in Canada, Ottawa, Science Council of Canada, 1981, e Bryan Schwartz, Public Inquiries, Canadian Public Administration 40 (1) 1997: 72-85.

${ }^{68}$ Ver Claus Mueller, The Politics of Communication: A Study in the Political Sociology of Language, Socialization and Legitimation, New York, Oxford University Press, 1973: 145.
} 


\section{Conclusão: \\ A natureza complexa das políticas públicas e da opinião pública nos estados democráticos contemporâneos}

A opinião pública, então, afeta apenas minimamente a formação de políticas? Ainda que sejam poucas as dúvidas de que o papel desempenhado pela opinião pública é muito menos direto do que sugerem os modelos lineares, isso não significa que é desimportante. A opinião pública é uma 'condição de fundo' importante, com base na qual a formação de políticas ocorre e depende. Além disso, o fato de que as ações governamentais precisam de legitimação nas sociedades democráticas assegura que a opinião pública continuará importante e será um fator levado em consideração pelos formadores das políticas, que poderão gastar muito tempo e esforço tentando administrála e manipulá-la. Trabalhos recentes de Kickert, Klijn, Koppenjan e outros, da 'Escola de Rotterdam', por exemplo, salientaram a maneira como os governos recorreram a um novo conjunto de instrumentos de políticas para conduzir a sociedade a fim de reter sua capacidade de influenciar os resultados das políticas. ${ }^{69}$ Trabalhos semelhantes no Reino Unido e na França por Rhodes, Marsh e outros estudaram problemas e perspectivas para "governança sem governo", tanto nacionalmente como dentro do sistema emergente na

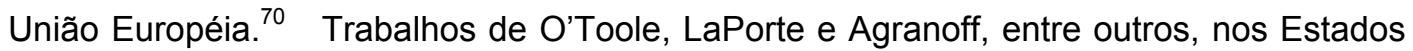
Unidos, também se ocuparam com diversas questões relacionadas à implementação efetiva de políticas numa era de "esvaziamento do estado". ${ }^{71}$ Todos esses trabalhos sublinham a natureza dialética da relação entre política pública e opinião pública, e a significação contínua, e mesmo crescente, da compreensão desta relação para os governos democráticos modernos e a governança.

Tradução do original em inglês de Plínio de Souza Dentzien

\footnotetext{
${ }^{69}$ Ver Erik-Hans Klijn, Analysing and Managing Policy Processes in Complex Networks: A Theoretical Examination of the Concept Policy Network and Its Problems, Administration and Society 28 (1) 1996: 90-119; Johan A. de Bruijn e Ernest F. tem Heuvelhof, Policy Networks and Governance, in David L. Weimer (org), Institutional Design, Boston, Kluwer Academic Publishers, 1995: 161-179; e de Bruijn, J. A., e E. F. tem Heuvelhof, Instruments for Network Management, in W. J. M. Kickert, E.-H. Klijn e J. F. M. Koppenjan (orgs), Managing Complex Networks: Strategies for the Public Sector, Londres, Sage, 1997: 119-136.

${ }^{70}$ Rhodes, R. A. W., Understanding Governance: Policy Networks, Governance, Reflexivity and Accountability, Buckingham, Open University Press, 1997; Marsh, David, Comparing Policy Networks, Buckingham, Open University Press, 1997; e P. Le Gales e M. Thatcher (orgs), Les Reseaux de Politique Publique, Paris, Editions L'Harmattan, 1995.

${ }^{71}$ Ver Milward H. Brinton, Keith G. Provan e Barbara A. Else, What does the 'Hollow State' Look Like? In B. Bozeman (org), Public Management: The State of the Art, San Francisco, Jossey-Bass, 1993: 309-323; Agranoff, Robert, e Michael McGuire, Managing in Network Settings, Policy Studies Review 16 (1) 1999: 18-41; O’Toole Jr., Laurence J., Implementing Public Innovations in Network Settings, Public Administration 29 (2) 1997: 115-139; O'Toole, Laurence J., Treating Networks Seriously: Practical and Research-Based Agendas in Public Administration, Public Administration Review 57 (1) 1997: 45-52; e La Porte, Todd R., Shifting Vantage and Conceptual Puzzles in Understanding Public Organization Networks, Journal of Public Administration Research and Theory 6 (1) 1996: 49-75.
} 